\title{
Los intangibles en el cambio de entidad de los clientes de banca detallista
}

\author{
Intangible Aspects when Detailed \\ Banking Customers Change Banks
}

\author{
José Pita Castelo (España) \\ Doctor en Ciencias Económicas y Empresariales \\ Universidad de Vigo \\ Correo electrónico: jpita@uvigo.es
}

\author{
Miguel González-Loureiro M. (España) \\ Doctor en Ciencias Económicas y Empresariales \\ Universidad de Vigo \\ Correo electrónico: mloureiro@uvigo.es
}

\section{Resumen \\ En este artículo se presentan \\ implicaciones de gestión respecto del cambio de entidad de los clientes en banca detallista, identificando dos tipos de dimensiones de satisfacción: tangibles e intangibles. Esta novedad permite analizar la relevancia de los intangibles sobre la lealtad. Para ello, se llevó a cabo una encuesta postal}

\begin{abstract}
This article presents management implications regarding the change of bank made by customers belonging to detailed banking. Two types of satisfaction dimensions are identified: tangible and intangible. This allows analyzing the relevance intangible traits have on loyalty. For this reason, we carried out a random survey on 384 users of detailed banking in
\end{abstract}

\begin{tabular}{|c|c|}
\hline $\begin{array}{l}\text { FECHA DE RECEPCIÓN: } \\
\text { FECHA DE REVISIÓN: } \\
\text { APROBACIÓN: }\end{array}$ & $\begin{array}{r}6 \text { de agosto de } 2014 \\
12 \text { de septiembre de } 2014 \\
16 \text { de septiembre de } 2014\end{array}$ \\
\hline
\end{tabular}

FECHA DE RECEPCIÓN: FECHA DE REVISIÓN: APROBACIÓN:
Para citar este artículo / to cite this article

Pita Caselo, J., González-Loureiro, M. y Duarte

Santos, J. (2014). Los intangibles en el cambio de entidad de los clientes de banca detallista. Poliantea, 10119), pp. 33-57. 
aleatoria simple a 384 usuarios de banca detallista en España, incluso cuestiones sobre calidad de servicio, satisfacción y probabilidad de cambio en caso de insatisfacción en cada una de las dimensiones de calidad de servicio. Se demuestra la existencia de una relación inversa entre satisfacción y comportamiento de cambio. Las dimensiones utilizadas profundizan en la valoración de la importancia de los aspectos intangibles frente a los tradicionales tangibles: los primeros proporcionan una mayor oportunidad para fidelizar clientes.

Palabras clave: satisfacción, competencia, banca detallista, cambio, lealtad, intangibles.
Spain, asking questions regarding quality of service, satisfaction, and possibility of change if there was some level of dissatisfaction. It was possible to show the relationship there is between satisfaction and change behavior. The dimensions used go into detail about the importance of intangible aspects with respect to the traditionally tangible ones. The former provide us with a better opportunity to foster loyalty among customers.
Keywords: Satisfaction, competition, detailed banking, change, loyalty, intangible. 


\section{Los intangibles en el cambio de entidad de los clientes de banca detallista}

\section{Intangible Aspects when Detailed Banking Customers Change Banks}

\author{
José Pita Castelo (España) \\ Doctor en Ciencias Económicas y Empresariales \\ Universidad de Vigo
}

Miguel González-Loureiro M. (España)

Universidad de Vigo

\section{José Duarte Santos (España) \\ Máster en Marketing \\ Instituto Superior Politécnico Gaya, Instituto Superior \\ de Contabilidade e Administração do Porto}

\section{Introducción}

El sector bancario español, desde 1990 a la actualidad, venía disfrutando de una alta lealtad de los clientes. Básicamente, un cliente no cambiaba de entidad salvo que cambiase de domicilio (Nielsen, 2011). Esta tendencia con probabilidad haya empeorado recientemente. La mayor competencia por conseguir clientes, en gran parte debido a cambios regulatorios y a una mayor concentración en el mercado bancario español (Salas y Saurina, 2003), ha conducido a una línea de estudios sobre la satisfacción con la calidad de servicio y lealtad de clientes en entidades financieras, contemplándose aspectos tangibles, como los costes de cambio (De Matos, Henrique Rosa, 2009), o intangibles, como la imagen corporativa (Bloemer, De Ruyter y Pascal, 1998), incluso contemplando las características sociodemógraficas de los clientes (Seiler, Rudolf y Krume, 2013). Sin embargo, pocos estudios 
han tratado expresamente sobre la influencia de la combinación de aspectos tangibles e intangibles sobre la lealtad (Serrano-Cinca, Mar Molinero y Chaparro, 2004).

Algunos autores sugieren que para medir el desempeño de estas entidades financieras es necesario ir más allá de las meras ratios financieras, $y$ que se debe indagar sobre creación de valor a través de cómo se presta el servicio y, específicamente, cómo se ensalza el valor de los intangibles (Cereceda, 1997). En el presente estudio, argumentamos que los aspectos intangibles, por su propia naturaleza inmaterial, son elementos sobre los que es más fácil construir la lealtad de clientes en la industria bancaria y defenderse del cambio de entidad que cuando dicha lealtad se construye sobre elementos tangibles.

Así, Aldás Manzano et al. (2011a) y Aldás Manzano et al. (2011b) analizan los determinantes de la lealtad en su caso de los servicios bancarios en línea. Los aspectos ahí incluidos son todos intangibles, como la confianza, la actitud, el riesgo percibido, el control percibido y la norma subjetiva. No obstante, subyace en dicho trabajo la idea de que la lealtad no varía en función de las dimensiones de servicio que se consideren. Este aspecto puede resultar ciertamente discutible, ya que supone implícitamente que el peso que tienen las dimensiones de servicio son las mismas siempre, independientemente del tipo de servicio y cliente. E indirectamente supone también la afirmación de que los atributos de producto no son relevantes para la lealtad o que incluso el mercado es único y no existen diferentes segmentos. Asimismo, no introduce variables tangibles relacionadas con el negocio financiero, por lo que su visión puede resultar relativamente incompleta al estar sesgada a dimensiones eminentemente intangibles.

En nuestro caso, planteamos la hipótesis de que la evaluación de la lealtad es un mix de aspectos tangibles e intangibles que pueden reforzarse mutuamente. Planteamos también que ambas, tangibles e intangibles, son dimensiones necesarias para la lealtad del cliente, pero no suficientes si alguna de ellas es excluida. Otras investigaciones han realizado caracterizaciones similares aplicadas en su caso al sector bancario en internet (Torres-Moraga, HidalgoCampos y Barra, 2008), pero poca investigación existe sobre la diferencia entre aspectos tangibles e intangibles en el conjunto del sector bancario. Diversas investigaciones vienen 
poniendo énfasis en el importante impacto que tienen los intangibles sobre el crecimiento de las empresas (González-Loureiro y Pita-Castelo, 2012; González-Loureiro y Figueroa Dorrego, 2012).

Así, se plantean las siguientes preguntas como guía de la investigación:

1. ¿Varía la relación entre satisfacción y cambio de entidad en función de la importancia de las dimensiones tangibles e intangibles del servicio? ¿Varía también en función de la similitud de ofertas de varias entidades respecto de una dimensión concreta?

2. ¿Hasta qué punto son determinantes las dimensiones tangibles financieras frente a las intangibles? En este caso, la categorización en dimensiones tangibles (básicamente financieras) e intangibles (fundamentalmente no financieros) es un aspecto novedoso de la investigación desde el punto de vista teórico y de actualidad entre los directivos de las entidades financieras.

Para dar respuesta a estos interrogantes, se introducen dos nuevas variables. Primero, la importancia de una determinada dimensión de servicio. Segundo, la naturaleza de la competencia se incluye como una dimensión de servicio más. En conjunto, se asume que estos factores son influyentes en el cambio de entidad, además de la variable satisfacción del cliente.

En la próxima sección, se desarrollan las hipótesis relacionadas con las dos preguntas guía de investigación mencionadas. Las hipótesis se comprueban mediante un estudio de campo entre residentes en España, en el que se emplea la encuesta como medio de recogida de información. Los detalles metodológicos del estudio empírico se presentan en la sección siguiente. Después se discuten los resultados y, finalmente, se proporcionan implicaciones para la gestión, así como las principales líneas de investigación futura según los hallazgos de esta investigación.

\section{Antecedentes e hipótesis}

En el tracking semestral al servicio de las entidades financieras Nielsen, realizado en España en el segundo semestre de 2010, uno de cada cuatro clientes trasladó al menos una cuenta bancaria a otra entidad durante los doce meses anteriores (Nielsen, 
2011). Las razones citadas para el traslado eran un mix de aspectos intangibles y tangibles. Entre los primeros estarían la disponibilidad o el mejor servicio; y entre los segundos, las menores comisiones y los tipos de interés más altos en depósitos. La investigación de Moutinho y Meidan (1989) sugiere que aumenta la probabilidad de que los clientes cambien de entidad bancaria si los costes de uso de las tarjetas de crédito son altos, si los intereses de los depósitos son bajos y si se deniegan solicitudes de préstamo.

Aunque una oferta financiera mejor (por lo tanto, aspectos tangibles-financieros) parece ser la razón principal para cambiar de entidad financiera, también la disponibilidad y el servicio (intangibles) parecen ser consideraciones válidas (Gwin y Lindgren, 1986). En resumen, un cliente puede pensar en cambiar de entidad financiera si no está satisfecho con su oferta por cualquiera de estos motivos: temas financieros, temas de servicio o temas de disponibilidad. En consecuencia, la hipótesis de que la satisfacción relacionada con cualquier servicio bancario está relacionada inversamente con la probabilidad de cambiar de entidad por ese servicio particular está ampliamente testada y apoyada por numerosos estudios no ya solo en el ámbito general de las relaciones de marketing, sino también en el bancario en particular. Estudios clásicos, como el de Reichheld y Kenny (1990), señalan que un incremento de escasamente $5 \%$ en la tasa de retención mejora el rendimiento de cualquier entidad financiera, incluso en los casos más problemáticos. Los temas de "cambio de entidad" y "lealtad bancaria" son, por consiguiente, de las principales preocupaciones del negocio financiero detallista. La mayor parte de las investigaciones relacionadas con estos temas se ha centrado en asuntos como la elección de entidad financiera (Anderson, Cox y Fulcher, 1976; Pita-Castelo, 2002), la banca de relaciones (Berry y Thompson, 1982; Esteban et al., 2000; Gómez, 2000), la segmentación de mercado (Kinnaird et al., 1984; Hood y Walters 1985) y cambio de entidad y lealtad bancaria (Jain, Pinson y Malhotra, 1987; Moutinho y Brownlie, 1989; Berné, Yagüe y Múgica, 1996).

Algunos otros se han derivado hacia la experiencia en banca en línea, cuyas características de prestación de servicios son evidentemente diferentes del ser detallistas sin establecimiento por definición, aun cuando 
algunos de ellos pueden llegar a usar diferentes marcas para los establecimientos que para la banca en línea (Herington y Weaven, 2007; Heinonen, 2007; Ho y Lin, 2010; Wong, Rexha y Phau, 2008). En este caso, las dimensiones evaluadas tienen que ver con las características propias de la no presencialidad en la prestación del servicio bancario (Eriksson y Nilsson, 2007; Zeng et al., 2009; Chau y Ngai, 2010).

Esta literatura sugiere que la insatisfacción con un servicio bancario concreto es una de las principales razones por las que los clientes cambian de entidad. La contribución principal del presente estudio es profundizar en el conocimiento de la relación satisfacción-insatisfacción y el cambio de entidad de los clientes. Adicionalmente, se pretende también profundizar en el conocimiento de la relevancia que algunos aspectos intangibles llegan a tener con respecto a los aspectos tangibles. Estos últimos suelen ser generalmente los determinantes en la elección de la entidad financiera, pero ¿regirá el mismo criterio en la lealtad de clientes? En este sentido, Serrano-Cinca, Mar Molinero y Chaparro (2004) ofrecen una perspectiva sobre intangibles en cajas de ahorro de España a partir de una categorización de intangibles a partir de los informes de capital intelectual de algunas entidades financieras. Sugieren también que para medir la performance de estas entidades financieras es necesario ir más allá de las meras ratios financieras e indagar en la manera en que crean valor con los intangibles.

\section{Importancia de los servicios bancarios}

Las personas responsables de los departamentos de marketing de las entidades financieras saben que no todos los servicios tienen la misma importancia para todos los clientes. En primer lugar, los clientes tienen distintos niveles de "sensibilidad vertical", es decir, la importancia de un servicio determinado para los distintos clientes no es la misma. Y en segundo lugar, los servicios tienen distintos niveles de "sensibilidad horizontal", es decir, la importancia de los diferentes servicios no es la misma para un cliente determinado. Así, "la importancia de un servicio concreto para un cliente concreto" es un factor crítico en la determinación de las respuestas de los clientes ante servicios no satisfactorios. Esto ha dado como resultado la propuesta de una metodología para medir "atributos 
determinantes", procedimiento que implica la medición de las actitudes hacia los atributos más cercanos a las decisiones de preferencia o de compra (Alpert, 1971; Myers y Alpert, 1968).

La técnica de análisis de atributos determinantes, definida en el estudio seminal de Krech y Crutchfeld (1948), ha sido aplicada en las decisiones de selección de entidad y también para identificar segmentos de consumidores. Uno de los objetivos de la presente investigación es aplicar este análisis de atributos determinantes para profundizar en el conocimiento del comportamiento de cambio de entidad. Cuanto más importante sea un atributo para un individuo, más determinante será en la decisión que tome.

Diversos estudios han destacado ya la positiva relación existente entre calidad de servicio percibida y satisfacción en la industria bancaria (Bloemer, De Ruyter y Peeter, 1998; Licata y Chakraborty, 2009; Valdunciel, Flórez y Dávila, 2007). Esto ha hecho que se haya centrado el estudio en definir las dimensiones de la calidad de servicio para su mejora (Valdunciel, Flórez y Dávila, 2007), pero se haya descuidado su posible impacto en un probable cambio de entidad bancaria.
Un conjunto de estudios de la calidad de servicio en la industria bancaria se basó en la consideración de la escala SERVQUAL de Parasuraman, Zeithaml y Berry $(1985,1988)$, tales como el de Yavas, Bilgin y Shemwell (1997), Bahia y Nantel (2000) o Fernández-Barcala (2000). En ellos se toman las habituales cinco dimensiones de tangibles, fiabilidad, capacidad de respuesta, seguridad y empatía. Otros estudios han utilizado la escala SERVPREF, que al contrario de la anterior, tiene en cuenta la diferencia existente entre la percepción y la importancia de las anteriores dimensiones (Sharma y Mehta, 2004; Bauer, Hammerschmidt y Falk, 2005). Otros estudios han desarrollado sus propias escalas de medición para la industria bancaria, tales como Aldlaigan y Buttle (2002), Al-Hawari, Hartley y Ward (2005) o Valdunciel, Flórez y Dávila (2007), quizás uno de los trabajos más integradores existentes sobre este aspecto.

Sin embargo, no es frecuente encontrar la idea de que la satisfacción y la calidad de servicio puedan estar moderadas por las dimensiones de servicio consideradas, en lugar de considerarse el servicio como único, tal y como se ha hecho en los trabajos citados. Sin embargo, en 
investigaciones desarrolladas sobre empresas de servicios, se ha observado la existencia de efectos mediadores múltiples, que implica que la relación entre satisfacción y lealtad están indirectamente influida por variables relativas al servicio en cuestión (Kantsperger y Kunz, 2010).

Asíla relación entre satisfacción con un servicio bancario determinado y la lealtad o probabilidad de cambiar a otra entidad por ese mismo servicio debiera mejorar si la importancia de ese servicio también es tenida en cuenta. Esto significa que la satisfacción con un servicio bancario explica el cambio de entidad solo hasta cierto punto, porque varía en función de las dimensiones de servicio que se consideren y de su importancia relativa. Así se hipotetiza que:

H1: la relación entre satisfacción y la probabilidad de cambiar a otra entidad es corregida por la importancia concedida a las dimensiones de servicio.

Se espera que el control de la importancia de las dimensiones de servicio resulte en relaciones más uniformes entre satisfacción y probabilidad de cambio de entidad a lo largo de las diferentes dimensiones de servicio.

\section{Similitud de ofertas de competitivas}

Es lógico suponer que si las ofertas competitivas son similares, es decir, no existe diferencia entre las ofertas competitivas de varias entidades competidoras, o dicha diferencia es ínfima, es poco probable que el cliente cambie a pesar de la importancia del servicio o su nivel de insatisfacción. Esto se debe a que el cliente percibe que no es probable que el cambio mejore su situación (Licata y Chakraborty, 2009). Por tanto, permanecerá en la misma entidad en vez de emplear esfuerzo y tiempo en el cambio de proveedor.

Mientras la importancia de un servicio particular para un cliente determinado representa "el factor de diferencia individual", la similitud de las ofertas competitivas representa "el factor de sistema” respecto de las opciones/alternativas disponibles para cambiar. Así, en las dimensiones tangibles-financieras de servicio bancario en las que las ofertas competitivas son similares, la relación entre satisfacción y cambio es menos probable que se vea afectada por la importancia de dicha dimensión de servicio (Serrano-Cinca, Mar Molinero y Chaparro, 2010), o incluso por la frecuencia de uso de dicho servicio (Aldás Manzano et al., 2011b). 
Es interesante observar tipos de interés similares en depósitos, cuentas corrientes, préstamos, etc., ofertados por entidades competidoras en una zona geográfica; en resumen, la posibilidad de que las entidades impongan costes de cambio que evite el cambio de entidad (De Matos, Luiz Henrique y De Rosa, 2009). En cambio, las dimensiones intangibles no financieras del servicio bancario como la actitud del personal, el aspecto de las instalaciones y el entorno, entre otros, probablemente se vean como independientes de las dimensiones tangibles financieras (Serrano-Cinca, Mar Molinero y Chaparro, 2004).

Aun así, las ofertas competitivas sobre estas dimensiones intangibles de servicio pueden ser más variables debido a la propia naturaleza intangible inherente en la prestación de un servicio (Serrano-Cinca, Mar Molinero y Chaparro, 2004; Aldás Manzano et al., 2011b). En resumen, es probable que la importancia de las dimensiones intangibles no financieras de servicio tengan mayor influencia sobre la relación entre satisfacción y probabilidad de cambio que la importancia de las dimensiones tangibles financieras.

Así, se hipotetiza que:

H2: la relación entre la insatisfacción y la probabilidad de cambio de entidad es mayor en las dimensiones intangibles - cuando se controla la importancia del servicio-que en las tangibles.

H3: La satisfacción con las dimensiones tangibles del servicio bancario no difiere entre las distintas entidades, mientras que en las dimensiones intangibles la satisfacción sí varía.

Es importante señalar aquí la importancia que suponen estas hipótesis desde la perspectiva de las estrategias competitivas para las entidades financieras. Equivale a decir que las dimensiones tangibles financieras del servicio bancario no parecen realmente determinantes en la diferenciación a largo plazo, puesto que la satisfacción en las dimensiones tangibles no difiere entre las entidades (H3). La otra cara de la moneda es que precisamente hay mayor probabilidad de cambio al comparar la oferta entre entidades según las dimensiones intangibles (implícito en $\mathrm{H} 2$ ), lo cual convertiría a las dimensiones intangibles como fundamentales. Esta hipótesis adquiere mayor importancia hoy en día si se tiene en cuenta la igualdad en las dimensiones tangibles financieras debido a las condiciones del mercado. 
Metodología del estudio empírico Variables y métodos

El cuestionario incluía preguntas para medir las tres dimensiones básicas incluidas en esta investigación, es decir, satisfacción e importancia y probabilidad de cambio a otra entidad en función de varios atributos de servicio bancario (lealtad). También se recogieron datos sobre información demográfica. Las escalas y preguntas específicas para medir estas tres dimensiones se describen a continuación y se sintetizan en la tabla 1 , en la que se proporciona una lista de los 20 atributos de servicio sobre los cuales se midieron la satisfacción del cliente y la importancia que le daban a cada atributo. Esta lista fue creada basándose en la bibliografía existente sobre marketing bancario (Bou y Camisón, 1999; García de Madariaga y Pita-Castelo, 2001; Pita-Castelo, 2002; Manrai y Manrai, 2007; Valdunciel, Flórez y Dávila, 2007; Ho y Lin, 2010), y la experiencia personal con entrevistas a directores de oficinas de una diversidad de tipos de entidades bancarias con establecimientos detallistas, realizadas entre enero y febrero de 2012. Entre ellas había seis entrevistados de cajas de ahorros (la antigua Caixanova, la antigua Caixagalicia y La Caixa), tres entrevistados de bancos con gran presencia comercial en toda España (Banco Santander, BBVA y Bankinter) y dos entrevistados de bancos con presencia minoritaria en España (Banco Popular y Banco Espírito Santo). Este último era también una representación de entidades bancarias extranjeras que operan en España.

Tabla 1. Lista de los atributos de servicio bancario utilizados para la medición de la satisfacción, la importancia y la probabilidad de cambio de entidad

\begin{tabular}{|l|l|}
\hline 1 & Aspecto de las instalaciones \\
\hline 2 & Aspecto del personal \\
\hline 3 & Horario de funcionamiento del cajero automático \\
\hline 4 & Actitud y comportamiento del personal \\
\hline 5 & Actitud y comportamiento de los clientes \\
\hline 6 & Competencia y eficacia del personal \\
\hline 7 & Decoración y ambiente de la entidad \\
\hline 8 & Horario de apertura entre semana \\
\hline 9 & Horario de apertura los sábados \\
\hline 10 & Tipos de interés en depósitos a la vista y a plazo fijo \\
\hline 11 & Tipos de interés en hipotecas \\
\hline 12 & Tipos de interés en planes de pensiones \\
\hline 13 & Tipos de interés en otros préstamos \\
\hline 14 & Tipos de interés en cuentas de ahorro \\
\hline 15 & Procedimientos para reclamaciones \\
\hline 16 & Fiabilidad del personal \\
\hline 17 & Respuesta a las necesidades del cliente \\
\hline 18 & Seguridad del entorno de la sucursal \\
\hline 19 & Tamaño de la sucursal \\
\hline 20 & Tiempo de espera en cajero automático \\
\hline & \\
\hline
\end{tabular}

A partir de este listado de atributos, la investigación realizada debe evaluar inicialmente si se pueden agrupar en factores tangibles e intangibles para testar posteriormente 
las hipótesis planteadas. La tabla 2 ofrece la información relativa a cómo dichos atributos convergen en los siguientes aspectos:

\section{Intangibles: en este caso se trata} de factores relativos a la profesionalidad, el servicio ofrecido con cajero automático y el servicio que ofrece el horario.

2. Tangibles: son factores relativos a las instalaciones físicas, los intereses acreedores y los intereses deudores.

Esta separación en atributos tangibles e intangibles es una contribución del presente estudio a otros desarrollados sobre satisfacción y lealtad en servicios bancarios (Valdunciel, Flórez y Dávila, 2007; Aldás Manzano et al., 2011a, 2011b).

La satisfacción en el presente estudio se conceptualiza como constructo multidimensional, siguiendo investigaciones anteriores (Bloemer, De Ruyter y Pascal Peeters, 1998; Licata y Chakraborty, 2009; Valdunciel, Flórez y Dávila, 2007). Se solicitaba a los entrevistados que otorgasen puntuaciones a su entidad financiera principal para cada uno de los atributos señalados en la tabla 1 , utilizando para eso una escala de Likert de satisfacción desde 1 como muy insatisfecho a 5 como muy satisfecho. También se utilizó el mismo conjunto de atributos para generar las puntuaciones de importancia. A los entrevistados se les solicitaba que esta vez indicasen la importancia de cada uno de aquellos atributos de servicio en una escala de importancia tipo Likert desde 1 nada importante a 5 muy importante y también se solicitó a los entrevistados que considerasen para cada atributo de servicio su probabilidad de cambiar a otra entidad financiera por insatisfacción con ese atributo de servicio concreto. La escala empleada tipo Likert fue de 1 muy poco probable que cambie a 5 muy probable que cambie.

En el cuestionario se incluyeron otras preguntas de control de tipo demográfico, como edad, sexo, estado civil, nivel de estudios, categoría profesional, empleo e ingresos.

Una primera etapa en el análisis de resultados se centró en la obtención de los factores o dimensiones del servicio bancario. Esto se realizó mediante un análisis de componentes principales siguiendo la técnica de autovalores mayores de uno y una posterior rotación Varimax con normalización siguiendo el método de Kaiser. Este método de componentes principales es el más 
adecuado cuando el objetivo es la reducción de datos en el supuesto de que existe una estructura subyacente entre las variables (Kline, 1994). Como medida de fiabilidad de los constructos se utilizó el alfa de Cronbach (Cronbach, 1951), que es definido como la proporción de variabilidad en las respuestas a la encuesta que se deben a diferencias en los sujetos encuestados. Los valores cercanos a 1 indicarán que el instrumento de medida es confiable en la medida en que es capaz de capturar diferentes opiniones, mientras que los valores bajos, menores de 0.5 o cercanos a 0 indicarían que la variabilidad se debe a que el instrumento de medida es confuso o puede tener múltiples interpretaciones.

Posteriormente, se obtuvieron los coeficientes correlación simple o de orden 0 (Pearson) de cada atributo de servicio respecto de la probabilidad de cambio y también los coeficientes correlación parcial de primer orden. Estos últimos se pueden interpretar como los estimadores correspondientes a la satisfacción en cada atributo de servicio en una regresión múltiple de la "probabilidad de cambio" sobre "satisfacción" una vez controlado o "descontado" el posible impacto que la "importancia" de una dimensión de servicio puede tener sobre la probabilidad de cambio. En el caso de que los coeficientes de correlación parcial de orden 1 sean significativos, entonces implicaría que la variable por la que se controla el efecto es significativa e influyente (Cohen, 1988).

Finalmente, para cada uno de los grandes factores de atributos tangibles e intangibles de servicio detectados, se realiza un análisis de varianza (Anova) o prueba comparativa entre entidades, el cual es una extensión del t-test para dos muestras (Brown y Forsythe, 1974b). Para el contraste de diferencias entre entidades, se utilizó el estadístico F de Brown-Forsythe (1974a, 1974b), el cual contrasta la hipótesis nula de que las medias sean iguales entre grupos de casos, por lo que un nivel de significación menor de 0.05 rechazará la hipótesis nula, lo que implica que la diferencia de medias es significativa.

\section{Muestra y trabajo de campo}

Se siguió un enfoque cuantitativo para la generalización de muestra a la población. Se envió entre abril y mayo de 2012 por correo postal a una muestra aleatoria simple de clientes bancarios latinoamericanos en España una carta en la que se solicitaba que respondieran al cuestionario que se adjuntaba y lo devolvieran por 
correo postal en el sobre prefranqueado que se les proporcionaba. La aplicación se realizó a través de una rutina informática en el centro del proceso de datos de un importante banco español con requerimiento de confidencialidad. Se enviaron 670 cuestionarios y se recibieron 384 que dieron una tasa de respuesta de $58 \%$, con un nivel de confianza de la muestra de $95 \%$ y un error de $5 \%$, asumiendo población infinita.

Todos los encuestados tenían en común trabajar con la misma entidad (en la que se realizó el muestreo) y con otras. Como media trabajaban con 2.2 entidades. Como características principales de la muestra se pueden señalar que $69.8 \%$ eran hombres. La muestra estaba formada por personas jóvenes, ya que $80.2 \%$ tenía menos de 39 años, $48.4 \%$ ingresaba menos de $€ 1200.00$ mensuales, y solo $19.3 \%$ de la muestra declaraba que tenía estudios universitarios. El examen de la experiencia bancaria a partir de estos datos reveló que $90 \%$ de los que responden a la encuesta tienen una entidad financiera principal. Como media utilizaban varios servicios financieros: $71 \%$ utilizaba una cuenta corriente; $75 \%$, una cuenta de ahorro; $39 \%$ tenía un depósito a plazo; y $50 \%$, un plan de ahorro.

\section{Resultados y discusión} Dimensiones del servicio bancario Los datos de satisfacción del cliente sobre los atributos de servicio fueron sometidos a análisis factorial para obtener las dimensiones del servicio bancario. Los detalles del resultado se ofrecen en la tabla 2. Se identificaron seis factores y dos dimensiones de satisfacción del cliente. Estas últimas son la dimensión de los intangibles, como la profesionalidad y la disponibilidad (cajero automático y horario), y la de los tangibles, como consideraciones financieras (intereses deudores e intereses acreedores) o instalaciones.

Los resultados mostrados en la tabla 2 proporcionan perspectivas interesantes sobre las dimensiones del servicio bancario. Primero, los atributos tangibles financieros del servicio bancario se valoran aparte del resto de los atributos (no financieros). Además, dentro de la categoría de los atributos financieros, los clientes distinguen entre intereses deudores e intereses acreedores, tal y como se desprende del análisis que sitúa los atributos correspondientes en dos factores separados. De manera similar, la dimensión de disponibilidad tiene dos factores, es decir, la relacionada con los cajeros automáticos y 
la relacionada con el horario de la entidad.

La varianza explicada por los distintos factores es una indicación del grado de variabilidad de la satisfacción que existe en la muestra con respecto al servicio bancario en los seis factores. Es decir, la varianza describe el rango de respuestas, o sea, el grado de dispersión de las respuestas de los clientes en cada uno de los seis factores considerados. La dimensión tangible, que incluye instalaciones físicas y los factores de intereses acreedores y deudores (consideraciones financieras), explica solamente $25.4 \%$ de la varianza en contraposición con los factores intangibles (no financieros), como profesionalidad, cajero automático y horario, que explican $48.4 \%$ de la varianza. Este resultado sugiere que hay mayor variabilidad de la satisfacción del cliente en dimensiones intangibles que en dimensiones tangibles.

Tabla 2. Factores de los atributos del servicio. Resultados del análisis factorial (autovalor > 1). Correlaciones parcial y simple (Pearson)

\begin{tabular}{|c|c|c|c|c|c|c|}
\hline Dimensión & Factor (alfa de Cronbach)* & Atributos & $\begin{array}{l}\text { Varianza } \\
\text { explicada }\end{array}$ & $\begin{array}{l}\text { Correl. } \\
\text { simple }^{* \star *}\end{array}$ & $\begin{array}{l}\text { Correl. } \\
\text { parcial| }^{* *+*}\end{array}$ & $\begin{array}{l}\text { Dif. entre } \\
\text { correl. }\end{array}$ \\
\hline \multirow{11}{*}{$\begin{array}{l}\text { Intangibles } \\
0.484\end{array}$} & \multirow{7}{*}{$\begin{array}{l}\text { Profesionalidad } \\
a=0.90\end{array}$} & 2 & \multirow{7}{*}{0.371} & -0.10 & -0.19 & 0.09 \\
\hline & & 4 & & 0.03 & -0.13 & 0.10 \\
\hline & & 5 & & -0.17 & -0.22 & 0.05 \\
\hline & & 6 & & -0.10 & -0.17 & 0.07 \\
\hline & & 15 & & -0.16 & -0.17 & 0.01 \\
\hline & & 16 & & -0.19 & -0.20 & 0.01 \\
\hline & & 17 & & -0.10 & -0.19 & 0.09 \\
\hline & \multirow{2}{*}{$\begin{array}{l}\text { Servicio ofrecido por cajero } \\
\text { automático } \\
a=0.79\end{array}$} & 3 & \multirow[b]{2}{*}{0.057} & 0.03 & -0.13 & 0.10 \\
\hline & & 20 & & -0.17 & -0.22 & 0.05 \\
\hline & \multirow{2}{*}{$\begin{array}{l}\text { Servicio derivado horario } \\
a=0.81\end{array}$} & 8 & \multirow{2}{*}{0.056} & -0.10 & -0.17 & 0.07 \\
\hline & & 9 & & -0.16 & -0.17 & 0.01 \\
\hline \multirow{9}{*}{$\begin{array}{l}\text { Tangibles } \\
0.254\end{array}$} & \multirow{4}{*}{$\begin{array}{l}\text { Instalaciones físicas } \\
a=0.89\end{array}$} & 1 & \multirow{4}{*}{0.125} & -0.19 & -0.20 & 0.01 \\
\hline & & 7 & & -0.10 & -0.19 & 0.09 \\
\hline & & 18 & & 0.03 & -0.13 & 0.10 \\
\hline & & 19 & & -0.17 & -0.22 & 0.05 \\
\hline & \multirow{3}{*}{$\begin{array}{l}\text { Intereses acreedores } \\
a=0.90\end{array}$} & 10 & \multirow{3}{*}{0.07} & -0.10 & -0.17 & 0.07 \\
\hline & & 12 & & -0.16 & -0.17 & 0.01 \\
\hline & & 14 & & -0.19 & -0.20 & 0.01 \\
\hline & \multirow{2}{*}{$\begin{array}{l}\text { Interés de los deudores } \\
a=0.87\end{array}$} & 11 & \multirow{2}{*}{0.059} & \multirow{2}{*}{-0.10} & \multirow{2}{*}{-0.19} & \multirow{2}{*}{0.09} \\
\hline & & 13 & & & & \\
\hline
\end{tabular}

* Medida de la fiabilidad del factor, aceptable si a $>0.7$.

** La estructura factorial fue hallada utilizando la rotación Varimax.

$* * *$ Todos los valores son significativos $(p<0.05)$.

$\star * * * p<0.01$. Correlaciones parciales de primer orden. Coeficientes $b$ correspondientes a satisfacción en una regresión múltiple de probabilidad de cambio sobre satisfacción e importancia de una dimensión de servicio. 
El primer factor incluye atributos relacionados con la fiabilidad, actitud, competencia y eficiencia del personal. Este primer factor denominado profesionalidad explica la mayor proporción de varianza $(37.1 \%$ de la varianza total). El siguiente factor en explicación de varianza es el que contiene los atributos relacionados con las instalaciones (por ejemplo su aspecto, tamaño de la sucursal, etc.), y este factor explica $12.5 \%$ de la varianza total. Los atributos relacionados con la disponibilidad del servicio bancario fueron capturados por dos factores: cajero automático y horario, que explican $11.3 \%$ de la varianza total.

De esta manera, el servicio general de un banco puede ser descrito respecto de seis factores: profesionalidad, instalaciones, intereses acreedores, intereses deudores, cajeros automáticos y horarios. Para cada uno de estos factores se construyen variables compuestas mediante la suma de las puntuaciones respuesta de la muestra sobre los atributos respectivos que cargan en un factor particular. Con ellos se realizó una correlación simple (Pearson) entre satisfacción y probabilidad de cambio de entidad. Estas correlaciones y sus significados estadísticos se proporcionan en la tabla 2.
Como se podía esperar, la satisfacción se correlaciona de manera negativa con la probabilidad de cambio.

\section{Efecto de la importancia sobre la relación entre satisfacción y la probabilidad de cambio} A continuación, se realizaron correlaciones parciales (Pearson) entre satisfacción y probabilidad de cambio controlando la importancia de los diferentes servicios. Estos resultados se presentan en la tabla 2. Como se puede observar, la relación entre satisfacción y probabilidad de cambio continúa siendo negativa, y además el grado de correlación y significatividad estadística mejora para cada uno de los seis factores de servicio. El control de la importancia de un servicio particular también estabiliza la relación entre satisfacción y probabilidad de cambio a lo largo de las distintas dimensiones de servicio. Cuando no se controla la importancia, los coeficientes de correlación tienen un rango entre +0.03 y -0.19 (correlación simple). Este rango se reduce cuando la importancia se controla, rango entre -0.13 y -0.22 (correlación parcial). Estos resultados proporcionan evidencia empírica que apoya el papel mediador de la importancia, tal y como se hipotetiza 
en $\mathrm{H} 1$, y sugiere que la satisfacción por sí misma no es suficiente para predecir la probabilidad de cambio, sino que la importancia del servicio también desempeña un papel crítico.

\section{Efecto de la importancia sobre la relación entre satisfacción y probabilidad de cambio por dimensiones de servicio}

La comparación de las correlaciones simples entre satisfacción y probabilidad de cambio y las correlaciones parciales entre estas dos variables después de controlar la importancia se ofrecen en la tabla 2. Estos resultados sugieren que el efecto de la importancia varía con las diferentes dimensiones de servicio. Para la dimensión tangible hay solo una pequeña diferencia entre la correlación parcial y simple. En contraposición, en la dimensión intangible el control de la importancia provoca una diferencia sustancial, como resulta evidente de la diferencia existente entre correlación simple y parcial. De esta manera, el efecto de la importancia sobre la relación entre satisfacción y probabilidad de cambio varía entre las diferentes dimensiones de servicio. Este hecho está en línea y apoya de manera empírica la afirmación vertida en la hipótesis H2.

\section{Satisfacción en función de}

\section{la dimensión de servicio}

La hipótesis $\mathrm{H} 3$ formula que la satisfacción con los aspectos tangibles del servicio de una entidad financiera no varía entre las entidades mientras que en los aspectos intangibles las puntuaciones de satisfacción varían entre las distintas entidades. Para comprobar esta hipótesis se compararon las puntuaciones de diferencia de satisfacción entre entidades utilizando el análisis de varianzas entre grupos (Anova). Los resultados de este análisis se exponen en la tabla 3. Como indican estos resultados, en la dimensión tangible, es decir, intereses acreedores, deudores e instalaciones, no hay diferencias significativas entre las entidades. Por otro lado, las entidades sí que difieren de manera significativa entre sus puntuaciones de satisfacción sobre dimensiones intangibles, como profesionalidad y comodidad, o sea, cajero automático y horario. De esta manera, se halla apoyo empírico para la hipótesis H3. 
Tabla 3: Diferencias en puntuaciones de satisfacción entre entidades (One-way ANOVA)

\begin{tabular}{|c|l|r|r|r|}
\hline Dimensión & \multicolumn{1}{|c|}{ Factor } & $\begin{array}{r}\text { Suma de } \\
\text { cuadrados } \\
\text { entre grupos }\end{array}$ & \multicolumn{1}{|c|}{$\mathrm{F}^{*}$} & p-valor \\
\hline \multirow{4}{*}{ Intangible } & Profesionalidad & 6.69 & 3.01 & 0.03 \\
\cline { 2 - 5 } & $\begin{array}{l}\text { Cajero } \\
\text { automático }\end{array}$ & 4.29 & 2.67 & 0.04 \\
\cline { 2 - 5 } & Horario & 69.29 & 17.83 & 0.00 \\
\hline \multirow{4}{*}{ Tangible } & Instalaciones & 10.67 & 6.11 & $0.52^{* *}$ \\
\cline { 2 - 5 } & $\begin{array}{l}\text { Intereses } \\
\text { acreedores }\end{array}$ & 2.05 & 1.25 & $0.64^{* *}$ \\
\cline { 2 - 5 } & $\begin{array}{l}\text { Intereses } \\
\text { deudores }\end{array}$ & 3.74 & 1.03 & $0.55^{* *}$ \\
\hline
\end{tabular}

* Los grados de libertad del estadístico F para cada dimensión de servicio son (1.4).

** No significativo para $p<0.05$.

\section{Implicaciones de gestión y líneas futuras de investigación}

Esta investigación realiza tres contribuciones importantes con implicaciones para la gestión sobre temas relacionados con los intangibles, el cambio de entidad financiera y la lealtad del cliente. La evidencia empírica aportada apunta a que las empresas de servicios bancarios tienen ante sí la oportunidad y el reto de gestionar los intangibles como elementos que les pueden ayudar a reducir el riesgo de cambio de entidad. No obstante, esta conclusión no está exenta de controversia, por lo que presentamos a continuación nuestras conclusiones para que futuras investigaciones contribuyan a este debate.
En primer lugar, se identifican dos dimensiones generales de satisfacción del cliente con los servicios bancarios: tangibles e intangibles. En las entidades incluidas en esta investigación, las ofertas competitivas eran claramente similares en opinión de los clientes en cuanto a consideraciones tangibles, como los tipos de interés. Sin embargo, en los otros tres factores (intangibles) se hallaron diferencias significativas entre las entidades.

En segundo lugar, los hallazgos de esta investigación sugieren que las personas responsables de marketing de las entidades financieras necesitan prestar mucha más atención a factores promocionales intangibles, como el personal, el servicio ofrecido con cajero automático y la comodidad que han venido prestando hasta ahora. Esto ayudaría a las entidades de una doble forma. Por un lado, permitiría diferenciar sus ofertas de acuerdo con la percepción de los clientes y aumentar tasas de retención o atracción. Por otro lado, dado que los aspectos intangibles son más difícilmente imitables, supone una defensa frente a la competencia, que en muchos casos puede también dificultar que los clientes puedan comparar ofertas entre competidores. 
En tercer lugar, los hallazgos sugieren que el conocimiento de la importancia de cada servicio en particular ayudaría al responsable de marketing a comprender el grado de influencia de la satisfacción en la decisión de los clientes de permanecer fieles o embarcarse en comportamientos de cambio de entidad, ya que la importancia de un servicio particular desempeña un papel crítico en esta decisión, además de su satisfacción. Similares conclusiones fueron también apuntadas por Khan y Kadir (2011) desde la perspectiva de que lo determinante en el comportamiento y las actitudes de los jóvenes usuarios de banca es la percepción que tienen de los diversos elementos relacionados con el servicio bancario. Chau y Ngai (2010) también llegaron a similares resultados en la percepción que se tiene de un servicio bancario en línea y actitudes comportamientos. Por consiguiente, no parece recomendable desarrollar estrategias comunes de marketing, sino específicas en función del servicio particular.

Finalmente, los hallazgos sugieren que, incluso, si un cliente está insatisfecho con un servicio y el servicio es extremadamente importante, aquel puede no cambiar a otra entidad si la oferta percibida de la competencia es intrínsecamente similar. Esto es especialmente cierto para las dimensiones tangibles del servicio bancario, en las que las ofertas competidoras son similares. Sin embargo, en las dimensiones intangibles, la relación entre la probabilidad de cambio y satisfacción está significativamente afectada por la importancia del servicio, ya que las ofertas percibidas por los clientes están diferenciadas. Por tanto, la naturaleza de la competencia en las dimensiones intangibles de un servicio bancario desempeña un papel determinante en la decisión de cambio.

\section{Líneas futuras de investigación}

Existen varias vías para investigaciones futuras, con campo para replicar este estudio utilizando muestras de clientes más amplias y en otras regiones geográficas y otras procedencias culturales. Además, dado el papel crítico que desempeña la importancia del servicio en la mediación del efecto de la satisfacción sobre la probabilidad de cambio en los intangibles, parece relevante profundizar en el conocimiento de los determinantes de dicha importancia. Tales esfuerzos se podrían centrar en el futuro en la identificación de los perfiles de cliente en términos demográficos, 
psicográficos y culturales, así como en el incremento de posibles variables intangibles. Otro posible futuro estudio puede ser analizar eventuales diferencias en la percepción de los aspectos tangibles e intangibles por personas físicas en comparación con las personas jurídicas.

\section{Referencias bibliográficas}

Aldás Manzano, J., Lassala Navarré, C., Ruiz Mafé, C. y Sanz Blas, S. (2011a). Análisis de los factores determinantes de la lealtad hacia los servicios bancarios online. Cuadernos de Economía y Dirección de la Empresa, 14(1), 26-39.

Aldás Manzano, J., Lassala Navarré, C., Ruiz Mafé, C. y Sanz Blas, S. (2011b). Internet banking loyalty: evaluating the role of trust, satisfaction, perceived risk and frequency of use. The Service Industries Journal, 31(7), 1165-1190.

Aldlaigan, A. y Buttle, F. (2002). SYSTRA-SQ: s new measure of bank service quality. International Journal of Service Industry Management, 13(4), 362-381.
Al-Hawari, M., Hartley, N. y Ward, T. (2005). Measuring banks' automated service quality: a confirmatory factor analysis approach. Marketing Bulletin, 16(1), 1-19.

Alpert, M. I. (1971). Identification of determinant attributes: a comparison of methods, Journal of Marketing Research, 8, 184-191.

Anderson, W. T. Jr., Cox, E. P. III y Fulcher, D. G. (1976). Bank selection decisions and market segmentation. Journal of Marketing, 40(1), 40-45.

Bahia, K. y Nantel, J. (2000). A reliable and valid measurement scale for the perceived service quality of banks. International Journal of Bank Marketing, 1(2), 84-81.

Bauer, H. H., Hammerschmidt, M. y Falk, T. (2005). Measuring the quality of e-banking portals. International Journal of Bank Marketing, 23(2), 153-175.

Berné, C., Yagüe, M. J. y Múgica, J. M. (1996). La gestión estratégica y los conceptos de calidad percibida, satisfacción del cliente y lealtad. Economía Industrial, 307, 63-74. 
Berry, L. L. y Thompson, T. W. (1982). Relationship banking: the art of turning customers into clients. Journal of Retail Banking, 4(2), 64-73.

Bloemer, J., Ruyter, K. de y Peeters, P. (1998). Investigating drivers of bank loyalty: the complex relationship between image, service quality and satisfaction. International Journal of Bank Marketing, 16(7), 276-286.

Bou, J. C. y Camisón, C. (1999). Características métricas de los modelos de medición de la calidad percibida: una comparación de los modelos SERPERF y EP. Revista de Economía y Empresa, 36(XIII), 11-35.

Brown, M. B. y Forsythe A. B. (1974a). The small sample behavior of some statistics which test the equality of several means. Technometrics, 16(1), 129-132.

Brown, M. B. y Forsythe A. B. (1974b). Robust tests for the equality of variances. Journal of the American Statistical Association, 69(346), 364-367.

Cereceda, J. I. (1997). La fidelización en banca. Investigación y marketing. Investigación y Marketing, 57, 62-64.
Chau, V. S. y Ngai, L. W. L. C. (2010). The youth market for internet banking services: perceptions, attitude and behavior. Journal of Services Marketing, 24(1), 42-60.

Cohen, J. (1988). Statistical power analysis for the behavioural sciences (2. ${ }^{a}$ ed.). Hillsdale, New Jersey: Lawrence Erlbaum Associates.

Cronbach, L. J. (1951). Coefficient alpha and the internal structure of tests. Psychometrika, 16(3), 297-334.

Eriksson, K. y Nilsson, D. (2007). Determinants of the continued use of self-service technology: the case of internet banking. Technovation, 27(4), 159-167.

Esteban, A., Millán, A., Molina, A. y Martín-Consuegra, D. (2000). Identificación de los beneficios para el consumidor del marketing relacional: el caso de las entidades financieras. Revista Europea de Dirección y Economía de la Empresa, 9(3), 147-156.

Fernández Barcala, M. (2000). Validación de SERVQUAL como instrumento de medida de la calidad de servicio bancario. Revista Europea de Dirección y Economía de la Empresa, 9(1), 57-70. 
García de Madariaga, J. y Pita-Castelo, J. (2001). Las claves de satisfacción del cliente bancario. Cuadernos de Información Económica, 165, 115-125.

Gómez, M. (2000). Banca de relaciones y banca de transacciones: un análisis de las decisiones de marketing relacional en la banca corporativa española desde la perspectiva de los recursos. Revista Europea de Dirección y Economía de la Empresa, 9(3), 157-170.

González-Loureiro, M. y Figueroa Dorrego, P. (2012). Intellectual capital and system of innovation: what really matters at innovative SMEs. Intangible Capital, 8(2), 239-274.

González-Loureiro, M. y Pita-Castelo, J. (2012). A model for assessing the contribution of innovative SMEs to economic growth: the intangible approach. Economics Letters, 116(3), 312-315.

Gwin, J. M. y Lindgren, J. H. Jr. (1986). Reaching the service-sensitive retail consumer. Journal of Retail Banking, $8(3), 36-42$.

Heinonen, K. (2007). Conceptualising online banking service value.
Journal of Financial Services Marketing, 12, 39-52.

Herington, C. y Weaven, S. (2007). Can banks improve customer relationships with high quality online services? Managing Service Quality, 17(4), 404-427.

Ho, C. B. y Lin, W.-C. (2010). Measuring the service quality of internet banking: scale development and validation. European Business Review, 22(1), 5-24.

Hood, J. M. y Walters, C. G. (1985). Banking on stablished customers. Journal of Retail Banking, 7(1), 35-40. Jain, A. R., Pinson, C. y Malhotra, N. R. (1987). Customer loyalty as a construct in the marketing of banking services. International Journal of Bank Marketing, 5(3), 49-72.

Kantsperger, R. y Kunz, W. H. (2010). Consumer trust in service companies: a multiple mediating analysis. Managing Service Quality, 20(1), 4-25.

Khan, N. y Kadir, S. L. S. A. (2011). The impact of perceived value dimension on satisfaction and behavior intention: young-adult consumers in banking industry. African Journal of 
Business Management, 5(16), 7055- Journal of Bank Marketing, 27(7), 7067. 506-523.

Kinnaird, D., Shaughnessy, K., Stru- $\quad$ Moutinho, L. y Brownlie, D. (1989). man, K. D. y Swinyard, W. R. (1984). C Customer satisfaction with bank serMarket segmentation of retail bank vices: a multidimensional space analservices: a model for management. ysis. International Journal of Bank Journal of Retail Banking, 6(3), 52-63. Marketing, 7(5), 23-27.

Kline, P. (1994). An easy guide to fac- Moutinho, L. y Meidan, A. (1989). tor analysis. Londres: Routledge.

Krech, D. y Crutchfeld, R. S. (1948). Theory and problems of social psychology. Nueva York: McGraw-Hill Books Co.

Licata, J. W., \& Chakraborty, G. (2009). The effects of stake, satisfaction, and switching on true loyalty: a financial services study. International journal of bank marketing, 27(4), 252-269.

Manrai, L. y Manrai, A. (2007). A field study of customers' switching behavior for bank services. Journal of Retailing and Consumer Services, 14(3), 208-215.

Matos, C. A. de, Luiz Henrique, J. y Rosa, F. de (2009). The different roles of switching costs on the satisfactionloyalty relationship. International Bank customers' perceptions, innovations and new technology. International Journal of Bank Marketing, 7(2), 22-27.

Myers, J. H. y Alpert, M. I. (1968). Determining buying attitudes: meaning and measurement. Journal of Marketing, 32, 13-20.

Nielsen (2011). Servicios bancarios $y$ lealtad de los clientes: un reporte de Nielsen sobre el consumidor a nivel global. Nielsen.

Licata, J. W. y Chakraborty, G. (2009). The effects of stake, satisfaction, and switching on true loyalty: a financial services study. International Journal of Bank Marketing, 27(4), 252-269.

Parasuraman, A., Zeithaml, V. A. y Berry, L. L. (1985). A conceptual model of service quality and its 
implications for future research. Journal of Marketing, 49(4), 41-50.

Parasuraman, A., Zeithaml, V. A. y Berry, L. L. (1988). SERVQUAL: a multiple-item scale for measuring consumer perceptions of service quality. Journal of Retailing, 64(1), 12-40.

Pita-Castelo, J. (2002). Factores de selección de entidad financiera entre los clientes de múltiples entidades en España. Cuadernos de Investigación Económica, 168, 113-122.

Reichheld, F. F. y Kenny, D. W. (1990). The hidden advantages of customer retentions. Journal of Retail Banking, 12(4), 19-23.

Salas, V. y Saurina, J. (2003). Deregulation, market power and risk behaviour in spanish banks. European Economic Review, 47(6), 1061-1075.

Seiler, V., Rudolf, M. y Krume, T. (2013). The influence of socio-demographic variables on customer satisfaction and loyalty in the private banking industry. International Journal of Bank Marketing, 31(4), 235-258.

Sharma, A. y Mehta, V. (2004). Service quality perceptions in financial services: a case study of banking services. Journal of Services Research, 4(2), 205-223.

Serrano-Cinca, C., Mar Molinero, C. y Chaparro, F. (2004). Spanish savings banks: a view on intangibles. Knowledge Management Research \& Practice, 2(2), 103-117.

Torres-Moraga, E., Hidalgo-Campos, P. y Barra, C. (2008). Determination and categorization of the satisfaction and dissatisfaction factors in the internet banking. Academia-Revista Latinoamericana de Administración, 41, 49-67.

Valdunciel, L., Flórez, M. y Dávila, J. (2007). Análisis de la calidad del servicio que prestan las entidades bancarias y su repercusión en la satisfacción del cliente y la lealtad hacia la entidad. Revista Asturiana de Economía, 38(1), 79-108.

Wong, D. H., Rexha, N. y Phau, I. (2008). Re-examining traditional service quality in an e-banking era. International Journal of Bank Marketing, 26(7), 526-545.

Yavas, U., Bilgin, Z. y Shemwell, D. (1997). Service quality in the banking sector in an emerging economy: a consumer survey. International 
Journal of Bank Marketing, 15(6), service satisfaction and their impacts 217-223. on behavioural intentions. Total QualZeng, F., Hu, Z., Chen, R. y Yang, ity Management \& Business ExcelZ. (2009). Determinants of online lence, 20(9), 953-969. 